\title{
Política y género en los programas legislativos de ARENA y el FMLN
}

Las diferencias entre las plataformas legislativas de ARENA y del FMLN saltan a la vista desde el primer momento. El "Programa legislativo 2000-2003" del Frente anuncia, desde la presentación, que cuenta con una estructura básica que intenta darle unidad y cohesión a las propuestas. El "Compromiso 2000. Por un país seguro y más humano" de ARENA, en cambio, se dedica a ensalzar al partido, enunciando que es el mejor de los posibles, el único que ha sido capaz de sacar al país de la crisis económica y de llevarlo por el camino inequívoco del desartollo. Tanto el FMLN como ARENA dedican las primeras partes de sus plataformas a enumerar los logros que, a su juicio, han conseguido a lo largo de su desempeño, pero mientras el partido en el gobierno se aboca a la autoalabanza, el Frente pretende mantener una visión crítica con respecto a la Asamblea Legislativa.

En un franco atentando contra la inteligencia de la opinión pública, ARENA inaugura su propuesta de gobierno legislativo atribuyéndose los logros que el país ha alcanzado en materia democrática. Frases como "hemos hecho profundos cambios en El Salvador: Reformamos nuestro sistema político, ahora todos tenemos libertad de expresión; se respetan las ideas; vivimos en democracia" rayan en el cinismo más perturbador. ¿Quién a estas alturas no ha sido testigo del profundo espíritu antidemocrático que caracteriza a los areneros? ¿Quién no sabe que muchos de los cortos pasos que ha dado el país en el proceso de institucionalización democrática se han dado a pesar de ARENA y no gracias a ese instituto político?
Enumerar cada uno de los ejemplos fehacientes de ello sería imposible. Baste sólo con recordar el pasado violento e intolerante de muchos de los fundadores y actuales líderes de ARENA - fundadores y líderes también de los escuadrones de la muerte en la época del conflicto armado-; el rígido autoritarismo con el que opera internamente ese partido - lo cual le ha costado una cantidad considerable de disidencias y enemistades en los últimos años-; las comentadas censuras del Ministro del Interior, Mario Acosta Oertel; o la negativa del presidente Flores a participar en un debate público cuando era candidato a la presidencia, con lo cual desmintió su compromiso con la libertad de expresión de la que hoy se vanagloria su partido.

Además de la "profunda reforma al sistema político" que se adjudica, ARENA enumera entre sus logros aspectos que nada tienen que ver con el trabajo legislativo y que, en todo caso, deberían atribuirse al ejecutivo. Estos son: la modernización del sistema educativo; la reconstrucción de calles, carreteras y puentes; la construcción de viviendas populares a nivel nacional y de pasos a desnivel en San Salvador; la inversión extranjera; la reforma al sistema de pensiones y la "reforma" al sistema de salud. El que resalte estos aspectos indica que ARENA continúa apostando a conseguir votos a favor del partido, en tanto institución, y no a favor de las personas que aspiran a convertirse en diputados. Nada se menciona acerca del desempeño de la fracción arenera en el pleno porque, en definitiva, eso tiene para la cúpula arenera poca relevancia. Se invita a la gente a votar por ARE- 
NA simplemente porque es ARENA, el partido al que el país le debe la "reconciliación", la "paz", la "libertad"; aquél que con el esfuerzo de todos conIribuirá a traer el "progreso".

ARENA termina su presentación enunciando los desafíos que el nuevo siglo supone para lodos los salvadoreños: lograr "un país seguro y más humano" — en lo cual hará especial énfasis a lo largo de toda la propuesta_, "crear las oportunidades para que más familias salgan de la pobreza" y "construir una sociedad [...] basada en la solidaridad, en el trabajo, en la verdad y la justicia". ¿Acaso hay algo en el modo de gobernar de ARENA que apunte hacia la superación de esos desafíos? es la pregunta por plantear a quien redactó ese documento. Siendo cada vez más obvio que la práctica de los gobernantes areneros contradice palmo a palmo su discurso, ¿cabe tomarse en serio este supuesto compromiso con valores como la justicia, la verdad y la solidaridad? ¿No se desvirtuará inmediatamente una plataforma gubernamental que habla de cosas que nada tienen que ver con lo que el partido es en realidad?

El diagnóstico que en su propuesta de gobierno legislativo efectúa el FMLN sobre la situación de la Asamblea es, sin duda, mucho más aterrizado y realista que el planteamiento de ARENA. Para empezar, se centra únicamente en aspectos de esa rama del Estado y reconoce de entrada el descrédito en el que ha caído el pleno tras haber traicionado las expectativas que se habían generado en torno a él. Está claro que al principal partido de oposición le queda más fácil denunciar los errores y alumbrar los puntos oscuros de la gestión gubernamental. En última instancia, son errores y oscuridades que, según su análisis, obedecen a "causas estructurales, emanan del predominio sobre el Estado, la economía, los medios de comunicación [...], el sistema político y jurídico, ejercido por una pequeñísima minoría con enorme poder económico".

Pero no hay que perder de vista que, aunque las denuncias y señalamientos que efectúa en contra de la clase dominante sean en parte ciertos, en virtud de ellos el Frente cae fácilmente en la tentación de evadir su parte de responsabilidad en la lamentable situación de la Asamblea. Si en reconocer que las cosas están mal está la virtud de su plataforma, en culpar exclusivamente al partido oficial de ese malestar encuentra tal plataforma su principal debilidad. Evitando asumirse como la segunda fuerza política del país, el FMLN se limita a hacer una leve autocrítica cuando menciona el traumático caso del Procurador de Derechos Humanos. En ese punto reconoce que "pese a nuestra importante y protagónica participación en las elecciones [...] para Corte Suprema de Justicia, Fiscalía, Procuraduría General y de Derechos Humanos, los resultados obtenidos estuvieron muy por debajo de las expectativas ciudadanas, especialmente en el proceso de elección del Procurador de los Derechos Humanos que es el que mayor costo político nos generó". Como puede verse, el reconocimiento de lo que ellos mismos han llamado en varias oportunidades un error es aquí bastante tibio y poco comprometido.

Otra diferencia notable entre la plataforma del FMLN y la de ARENA -que nuevamente arroja un halance positivo de la primera respecto la segunda - es el hecho de que los logros que el Frenle se atribuye corresponden, en mucha mayor medida, a la realidad que los "profundos cambios al sistema político" que se adjudican los areneros. Se trata de una enumeración de aciertos todavía bastante general y cuestionable en varios puntos, de los cuales quizá el más sobresaliente sea el que resalta "la unidad y coherencia de nuestra fracción que ha permilido un mejor desempeño en las comisiones y pleno lcgislativo". Se sabe de sobra que esa "unidad y coherencia" se ha dado más por conveniencia que gracias al convencimiento sincero de las partes que continuamente se disputan el mando del partido - la última muestra de la pugna interna se dio durante el problemático proceso de elección del Fiscal-. Pero, aun así, los méritos que el FMLN reconoce como suyos al menos guardan relación con las cosas con las que siempre ha dicho estar comprometido y con las que en algunos casos ha demostrado estar dispuesto a defender. La rotunda negativa a la privatización es un ejemplo de ello.

A continuación se analizarán dos aspectos específicos de los programas legislativos de ARENA y del FMLN, con el propósito de precisar la valoración general que se ha hecho hasta ahora y de evaluar las ofertas de los partidos mayoritarios en aspectos particularmente relevantes de la problemática nacional. Tales aspectos son el tema político y las propuestas en materia de género.

\section{El tema político en las plataformas legislativas}

Consecuente con su convencimiento de que en El Salvador reina el Estado de Derecho y de que 
la democracia es una realidad que el país debe agradeccrle, ARENA plantea en su "Programa Legislativo" unas propuestas en materia política que resultan aún más pohres que las demás. En el apartado "Compromiso con la participación ciudadana" propone: "impulsar el proceso de modernización de la Asamblea Legislativa $|\ldots|$ requerimos una Asamblea que le asegure al pueblo una representación política y parlicipación electiva; a través de discusiones abierlas y transparentes que acerquen al pucblo a las decisiones. Requerimos una Asamblea ágil, moderna, eficiente, eficaz, cuya principal manifestación scan las respuestas oportunas a demandas de la población [...] Es necesario implementar medidas que: fortalezcan la Contraloría Social, que los ciudadanos supervisen el cumplimiento de los deberes de los funcionario públicos, desarroIlándose programas educativos cívico-democráticos, comunicacionales y de información".

Como puede verse, estos planteamientos no son sólo vaguedades y perogrulladas, sino que, nuevamente, ponen en evidencia las profundas contradicciones del discurso arenero con su práctica política. En primer lugar, no es cierto que a ARENA le interese escuchar y satisfacer las demandas de la población; su gestión se ha caracterizado más bien por desarrollarse de espaldas a esa demandas. En segundo lugar, tampoco es verdad que la fracción de ese partido procure la transparencia del pleno; es un secreto a voces que entre los diputados areneros las componendas, el amiguismo, la compra de votos y otras prácticas corruptas son una costumbre bien arraigada. Y, por último, resulta francamente hipócrita por parte de ARENA proponer el fortalecimiento de la "Contraloría Social", cuando la fracción legislativa de ese partido ha mostrado un especial desinterés en consolidar las instituciones contraloras y, por el contrario, ha contribuido grandemente a la precaria situación en la que éstas se encuentran. De nuevo, hay que decir que nada en el modo de gobernar de ARENA evidencia un compromiso real con la participación de la ciudadanía. Esa participación se da a su pesar y no favorecida por él. Y cuando se da, a los areneros -lejos de valorarla como un avance en la democratización del país- les representa un estorbo.

En el "Programa Legislativo" del FMLN pueden distinguirse al menos tres apartados en los que se aborda la temática política: (a) "Descentralización municipal", que apunta al fortalecimiento político e institucional de las municipalidades y a su democratización interna; $(b)$ "Asamblea Legislativa al scrvicio de la gente", constituido por propuestas destinadas a garantizar una mayor autoridad y autonomía a la Asamblea, acercar a los diputados a la comunidad, fortalecer la participación ciudadana, agilizar el trabajo en el pleno e impulsar un código de Ética Parlamentaria, entre otras; y (c) "Reestructuración del Estado", donde, básicamente, se proponen: el establecimiento y fortalecimiento de las contralorías públicas que contrarresten la corrupción y discrecionalidad de los funcionarios gubernamentales, el fortalecimiento de las obligaciones sociales del Estado, el incentivo a la participación ciudadana en aspectos políticos decisivos y la reestructuración del sistema judicial y del ejecutivo presidencialista.

Ciertamente, estas propuestas son mucho más concretas que el párrafo citado arriba en el que ARENA, más que plantear una oferta en el tema político, parecería querer convencer a los lectores sobre la importancia de la participación ciudadana y la transparencia en el manejo de la cosa pública. El FMLN apunta a problemas específicos del país que indudablemente urgen de respuestas eficaces. No obstante, su plataforma cae en el clásico error en el que suelen caer este tipo de documentos: no explica los procedimientos que permitirían llevar a cabo lo propuesto. Democratizar las alcaldías, 
aproximar la Asamblea a la ciudadanía y reestructurar el Estado son tareas importantes, necesarias e incluso realizables pero, ¿cómo van a efectuarse?, ¿qué mecanismos habría que seguir para que se hicieran realidad?, ¿puede garantizar el Frente que luchará por ellas hasta las últimas consecuencias?

Esta última pregunta está directamente relacionada con otra de las trabas que presenta al programa legislativo efemelenista: el desempeño de ese partido en el pleno durante el período que está por concluir. Muy atinados pueden ser el diagnóstico y la orientación que el FMLN da a su oferta política, pero mientras no termine de resolver sus pugnas intestinas, de trazar una línea ideológica clara, de renunciar tajantemente a prestarse a los juegos sucios que predominan en la Asamblea a la hora de resolver los problemas y de abrirse a concertar y a dialogar de modo que su tarea como oposición no parezca necia, sus propuestas carecerán de credibilidad. Y se trata de la credibilidad que le hace falta para conseguir los votos del gran número de desencantados que hacen mayoría entre la población en capacidad de votar.

La plataforma legislativa de ARENA indigna por cínica; la del FMLN, en cambio, parece bienintencionada y se ajusta más a las necesidades del país, pero es poco convincente porque deja de lado las propias taras del partido. Los areneros no hacen si no darle continuidad a su idea de que en el país todo está bien, que todo debe seguir como está y que sólo hacen falta unos leves ajustes para que las cosas marchen mejor. Eso es desesperanzador porque sin reconocer los errores es ilusorio esperar que se hagan las transformaciones necesarias. Así las cosas, de los diputados de ARENA no cabe más que esperar lo mismo. El Frente denuncia como siempre y sus denuncias aciertan, pero parece olvidar que él es parte fundamental del sistcma político al que critica y que debe reconocerse como tal a la hora de evaluarlo y de apuntar hacia las posibles soluciones que lo aquejan.

\section{El problema de género en las plataformas legis- lativas}

Al momento de evaluar y discutir el contenido de las plataformas legislativas se debe abrir un espacio para destacar el apartado dedicado a la problemática de género. Ya se sabe que cualquier propuesta o iniciativa que los mandatarios tomen a favor de la comunidad salvadoreña beneficia tanto a hombres como a mujeres, pero estas últimas tienen problemáticas particulares por solucionar. El tema de género se ha convertido en parte de la discusión de los planes y medidas gubernamentales, tras las constantes evidencias del menoscabo en que viven las salvadoreñas y la necesidad de reconocer tal menoscabo como la raíz de un cúmulo de injusticias que afectan el equilibrio social. En mucha medida, los problemas y dificultades a los que se enfrentan las mujeres responden a fallos y descuidos del sistema legislativo, que no ha sabido responder a sus necesidades. La Asamblea Legislativa es un espacio clave desde el cual impulsar soluciones que, cuando menos, logren mitigar las condiciones de marginación en las que permanece la población femenina.

El hecho de que los partidos políticos necesiten el voto de las mujeres, quienes constituyen el 52 por ciento de la población, y de que el sector femenino esté urgido de respuestas inmediatas de los gobiernos y personas al mando, debería aprovecharse como una condición favorable de la cual las mujeres podrían sacar provecho. Los políticos en contienda ofrecen a las votantes, además de soluciones a algunos de sus problemas, espacios de representación dentro de los círculos de poder, a cambio de su apoyo político. Esta necesidad mutua podría ser sana en tanto ambas partes pudieran salir beneliciadas. Desde el punto de vista de los problemas de género, estos beneficios tendrían que hacerse visibles en hechos, pero, lamentablemente, mucho de lo que las plataformas políticas plantean se queda en simples palabras. Las anteriores experiencias han dejado en el historial de la política salvadoreña una secuela de mentiras, falsos compromisos, palabras en el aire y una mayoría de votantes desencantados. Leer los planteamientos de las plataformas partidarias lleva siempre a preguntarse, ¿cuánto de lo que ahí se promete será una realidad dentro de tres años?

Ahora bien, tomando en cuenta que el primer paso para acabar con los conflictos es sacarlos a flote, el que exista en los programas legislativos un apartado dedicado especialmente a las mujeres puede ser considerado como un avance en la comprensión y compromiso de los políticos con los problemas de género. Supone el reconocimiento del sector femenino como un caso específico, que requiere proyectos y acciones particulares. De aquí a que lo expuesto se haga realidad falta un largo trecho, pero empezar a plantearlo es un buen inicio. 
Es necesario aclarar que existen diferencias sustanciales y evidentes entre las plataformas políticas a considerar. ARENA y el FMLN conciben de manera distinta la problemática de género y la forma de abordarla. Al parecer, el Frente entiende este fenómeno de un modo más amplio, más cercano a lo que el problema es en realidad. Por el contrario, la forma en que las mujeres son tomadas en cuenta dentro de la plataforma de ARENA es bastante más simplista, tendiendo a minimizar los problemas. Esta diferencia se ha visto también en anteriores plataformas, los planes de gobierno presidenciales en 1999 son el último ejemplo. Para entender de lo que se trata la visión de género dentro de los programas legislativos, se exponen a continuación las secciones que cada partido mayoritario a otorgado a las mujeres y los proyectos a realizar para los próximos tres años.

ARENA desglosa su plataforma en cuatro compromisos, de los cuales sólo dos incluyen a las mujeres. El primero es el "Compromiso con la Seguridad" que cuenta con una sección titulada "Seguridad Familiar". Es aquí donde se menciona por primera vez un compromiso de género. Fortalecer la familia, generar oportunidades de desarrollo para la mujer y evitar la violencia intrafamiliar es lo que los diputados areneros proponen. Más adelante, dentro del "Compromiso con la Participación Ciudadana", se encuentra el subtítulo "Desarrollo integral para población vulnerable", en donde se toman en cuentan a las mujeres jefas de hogar. Aquí, ARENA expone el proyecto de fortalecer "las normas legales que faciliten el acceso de las mujeres en el mercado laboral y el respeto a su integridad física y moral". Cabe mencionar que, según ARENA, son especílicamente las mujeres jefas de hogar las que necesitan estos beneficios y, además, se encuenIran caracterizadas de igual forma que las personas discapacitadas, los niños y jóvenes desprotegidos.

Como se puede ver, es muy poco lo que ARENA ofrece específicamente a las mujeres. De 20 proyectos generales que ARENA ha planilicado, to anterior es lo único que identifica el problema de género. Además, es necesario destacar que hay graves defectos dentro de estos compromisos. En principio, preocupa encontrar ideas tan generales y planteamientos tan poco depurados. Es obvio que con un bosquejo tan simple, las soluciones y acciones serán igualmente insuficientes. El asunto de género tiene muchas más aristas, y debe ser incluido también en los planes económicos, de seguri- dad pública, de salud, educación, del sector rural, etc. Por otra parte, al referirse a las mujeres jefas de hogar, se realiza una clasificación reduccionista y estereotipada, al concebir al sector femenino como débil víctima a la que hay que asistir y proteger. Aparentemente, ARENA ha disminuido el concepto y la labor de las mujeres a "jefas de hogar" y ha dejado de lado todas las demás funciones y necesidades del sector femenino. Esto deja entrever que los areneros poseen una valoración muy pobre sobre las mujeres, sus roles, derechos y obligaciones sociales.

El FMLN, por el contrario, ha dedicado una sección especial a las mujeres, elaborada a partir de los siguientes diez objetivos: (1) igualdad de participación de las mujeres en las decisiones políticas; (2) liderazgo y poder de las mujeres en las decisiones políticas; (3) que la nueva Constitución y Leyes se formulen con perspectiva de género $y$, donde sea pertinente, se especifiquen las acciones de las mujeres de los sectores urbano y rural; (4) que las leyes sean justas, no discriminatorias ni androcéntricas y que ningún sexo sea paradigma de otro; (5) que en el área de salud haya programas específicos para mujeres fuera del período productivo: niñas, mujeres maduras y ancianas; (6) que en el Código de Trabajo se visualice a las mujeres rurales y tengan acceso al trabajo con salarios equitativos y seguridad laboral; (7) hacer efectivo el compromiso del Gobiemo de El Salvador en Beijing: "facilitar la formulación y aplicación de políticas gubernamentales sobre igualdad entre mujeres $y$ hombres"; (8) ejecutar ese otro compromiso del Gobiemo: "reducir los efectos negativos de la crisis económica en las mujeres mediante programas y préstamos para el desarrollo social"; (9) hacer efectivo el compromiso de Santiago de Chile de 1994 con la "Convención interamericana para prevenir, sancionar y erradicar la violencia contra la mujer"; y (10) generar acciones y orientar el gasto social, de acuerdo con las necesidades de la población.

Lo primero a rescatar del proyecto de género del FMLN es el que se hayan tocado puntos más concretos. A su ve $z$, el abordaje de diferentes aspectos indica una concepción de las mujeres más integral; aporta más ideas y soluciones; se ve un proyecto más elaborado en pro de aquéllas. Lo más sobresaliente es quizás la identificación de diferentes problemas que se relacionan con los distintos roles de la población femenina. Por ejemplo, al disponerse a crear un 35 por ciento de espacios 
para diputadas se está detectando el machismo que siempre ha imperado dentro de la Asamblea Legislativa - recordando que 70 curules son ocupados por hombres y sólo 14 pertenecen a mujeres-. Asimismo, es importante el propósito de abrir más espacios de representación para éstas. Otro punto favorable es el reconocimiento de los vacíos legales respecto a las mujeres-. En general, puede decirse que si la población femenina votara únicamente por lo que se les ofrece, el FMLN llevaría la delantera. Aunque la plataforma del Frente resulta también insuficiente en muchos aspectos, no cabe duda de que este partido político tiene un aporte leórico más evolucionado y valioso en lo que se refiere al problema del género. Sólo queda una pregunta por hacer a los futuros diputados efemelenistas: de haber una jerarquización o criterio de selección al momento de ejecutar los planes que plantean en su plataforma, ia qué grado de protagonismo pueden aspirar las mujeres?

\section{Carmen Elena Villacorta Claudia Huete Guzmán}

